\title{
Metallo-Beta-Lactamase-Producing Acinetobacter spp. from Clinical Isolates at a Tertiary Care Hospital in Ghana
}

\author{
Michael A. Olu-Taiwo $\mathbb{D}^{1}{ }^{1}$ Japheth A. Opintan, ${ }^{2}$ Francis Samuel Codjoe, ${ }^{1}$ \\ and Akua Obeng Forson $\mathbb{D}^{1}$ \\ ${ }^{1}$ Department of Medical Laboratory Science, School of Biomedical and Allied Health Sciences, College of Health Sciences, \\ University of Ghana, Legon Accra, Ghana \\ ${ }^{2}$ Department of Medical Microbiology, Ghana Medical School, College of Health Sciences, University of Ghana, Legon Accra, Ghana
}

Correspondence should be addressed to Akua Obeng Forson; obeng.akua@yahoo.com

Received 20 May 2020; Accepted 24 August 2020; Published 25 September 2020

Academic Editor: György Schneider

Copyright (C) 2020 Michael A. Olu-Taiwo et al. This is an open access article distributed under the Creative Commons Attribution License, which permits unrestricted use, distribution, and reproduction in any medium, provided the original work is properly cited.

\begin{abstract}
Metallo-beta-lactamase-producing Acinetobacter spp. is a major challenge for therapeutic treatment of nosocomial infections. This study is aimed at determining the prevalence of MBL-producing Acinetobacter spp. among 87 clinical isolates of Acinetobacter spp. from the Korle-Bu Teaching Hospital, Accra, between August 2014 and July 2015. Acinetobacter spp. was identified by standard bacteriological method, and resistance to different antibiotics was assessed with the Kirby-Bauer disc diffusion method. Meropenem-resistant Acinetobacter isolates were screened for enzyme activity using the modified Hodge test (MHT) and combined disc test (CDT). Additionally, multiplex PCR was used to determine MBL genes presence (blaVIMblaIMP, and blaNDM). All Acinetobacter isolates showed high resistance to cefotaxime (90.8\%), ceftazidime (75.9\%), cotrimoxazole (70.1\%), ciprofloxacin (64.4\%), gentamicin (72.4\%), levofloxacin (67.8\%), and meropenem (59.8\%). A total of 54 (62.1\%) of Acinetobacter isolates were multidrug-resistant. Out of 52 (59.8\%) meropenem-resistant Acinetobacter, 3 (5.8\%) were carbapenemase producers by MHT, whilst, 23 (44.2\%) were CDT positive. There was no significant difference between the resistance pattern of amikacin, ceftazidime, cotrimoxazole, ciprofloxacin, and meropenem amongst CDT-positive and CDT-negative isolates $(p>0.05)$. A total of 7/87 (8.1\%) CDT-positive Acinetobacter isolates harboured blaNDM; of these, 4 (57.1\%) were from wound swabs, urine $(n=2)(28.6 \%)$, and ear swab $(n=1)(14.3 \%)$. The study revealed that less than $9 \%$ of Acinetobacter spp. contained blaNDM encoding genes. Strict antibiotics usage plan and infection control measures are required to prevent the spread of these resistance genes.
\end{abstract}

\section{Introduction}

In recent times, Acinetobacter spp. has been implicated in nosocomial infections of clinical importance in the elderly, infants, and immune-compromised patients [1-3]. Infections with Acinetobacter spp. lead to high mortality and morbidity, prolonged hospital stays with increased treatment costs. Carbapenem (meropenem and imipenem) are among the last resort for the treatment of serious Gram-negative bacilli infections [4]; however, carbapenem-resistant Acinetobacter has been on the increase to such an extent that carbapenem-resistant $A$. baumannii has been enlisted as one of the top priority pathogens by World Health Organiza- tion in 2017 [5]. Resistance to carbapenem could evolve by the development of efflux pumps, decreased cell permeability, and by the production of intrinsic or acquired carbapenemases belonging to either the class $\mathrm{B}$ or class $\mathrm{D}$ oxacillinases [6-8].

The emergence and dissemination of metallo-beta-lactamase- (MBL-) producing Acinetobacter spp. have become an important public health issue globally [4]. In Iraq, 56.6\% of Acinetobacter spp. was reported to be phenotypic MBL producers, of which, $10.73 \%$ and $2.8 \%$ harboured blaIMP and blaVIM [9]. In Egypt, a recent study reported $86.7 \%$ of A. baumannii was phenotypic MBL producers, whilst a similar study in Egypt reported $34.6 \%$ phenotypic MBL in $A$. 
baumannii, 23.07\% harboured blaVIM and blaIMP [10, 11]. In South Africa, $45 \%$ of $A$. baumannii was reported to be phenotypic MBL producers [12]. A previous study by Codjoe et al. [13] in Ghana reported a $14.4 \%$ prevalence for blaNDM in Gram-negative bacilli; however, the study's focus was on Gram-negative bacilli in general and not on the prevalence of MBL encoding genes in Acinetobacter spp. Limited information is available on carbapenemase activity in clinical isolates in Ghana; therefore, it is important to investigate MBL producers within the health-care settings, as early detection is critical for the effective formulation of antibiotic guidelines as well as the implementation of infection control strategies. This study is aimed at determining phenotypically and genotypically the prevalence of MBL encoding genes (blaIMP, blaVIM, and blaNDM) in carbapenem-resistant Acinetobacter spp. in a tertiary care hospital in the Accra metropolis.

\section{Materials and Methods}

2.1. Study Design and Site. This was a retrospective study of routinely collected clinical isolates of Acinetobacter spp. from Korle-Bu Teaching Hospital (KBTH). Korle-Bu Teaching Hospital (KBTH) is one of the largest health care facilities located in Accra, and it serves to provide health care for all categories of persons in Ghana [14]. It has more than 2000bed capacity with intensive care units that cater for surgical, medical, and trauma emergencies. It serves a paediatric and adult population of over 3 million in the Greater Accra region and acts as a major referral health facility for an estimated population of 22 million people across Ghana [14].

2.2. Acinetobacter Isolates. This study used clinical isolates of the genus Acinetobacter spp. recovered consecutively from 16000 clinical samples that included aspirates, eye swabs, ear swabs, wound swabs, and urine submitted to the Central Microbiology Laboratory of KBTH from August 2014 to July 2015. A total of 87 nonduplicate Acinetobacter isolates identified as causative agents of infection were inoculated into brain heart infusion broth (Difco/BD Diagnostic systems, Sparks, Michigan, USA) supplemented with 30\% glycerol and stored at $-80^{\circ} \mathrm{C}$ freezer at the Department of Medical Microbiology, University of Ghana Medical School, for subsequent tests and analyses. The clinical isolates were identified as per standard bacteriological methods using Gram stain, Triple sugar iron (TSI) fermentation test, and Oxidase, Indole, and Citrate utilization test as described by [15].

2.3. Antimicrobial Susceptibility Test for Acinetobacter spp. Isolates were subjected to Kirby-Bauer disc diffusion sensitivity testing per guidelines of the Clinical and Laboratory Standard Institute (CLSI) with Mueller-Hinton Agar [16] and EUCAST [17]. The following antibiotics purchased from Thermo Scientific ${ }^{\mathrm{TM}}$ Oxoid (United Kingdom) were used: amikacin $(30 \mu \mathrm{g})$, gentamicin $(10 \mu \mathrm{g})$, ampicillin $(10 \mu \mathrm{g})$, ciprofloxacin $(5 \mu \mathrm{g})$, levofloxacin $(5 \mu \mathrm{g})$, meropenem $(10 \mu \mathrm{g})$, amoxicillin/clavulanate $(30 \mu \mathrm{g})$, cefuroxime $(30 \mu \mathrm{g})$, ceftazidime $(30 \mu \mathrm{g})$, cotrimoxazole $(25 \mu \mathrm{g})$, and nitrofurantoin $(300 \mu \mathrm{g})$. Acinetobacter isolates with reduced susceptibility to meropenem $(10 \mu \mathrm{g})$ antibiotic disc (inhibition zone diameter $\leq 14 \mathrm{~mm}$ ) were selected for carbapenemase and Metallo-beta-lactamase detection (modified Hodge test and combined disc test) as described by Lee et al. [18] and Yong et al. [19]. Pseudomonas aeroginosa ATCC 27853 and Escherichia coli ATCC 25922 were used as control strains for quality control. According to the international standard definition for acquired resistance, multidrug-resistant (MDR) phenotype was defined as in vitro nonsusceptibility to at least one agent in three or more categories of antimicrobials [20].

2.4. Phenotypic Carbapenemase Screening Methods. All the carbapenem-resistant Acinetobacter isolates were screened for carbapenemase activity by the modified Hodge and combined disc methods. The modified Hodge test (MHT) was performed as previously described by Lee et al. [18]. Briefly, a control strain of Escherichia coli (ATCC 25922) adjusted to $0.5 \mathrm{McF}$ arland turbidity standard of inoculum was inoculated onto Mueller-Hinton (MH) agar (Oxoid, UK) as recommended by CLSI guidelines [16] and the plates were allowed to dry for 5 mins; then, one imipenem disc (10ug) (Oxoid, UK) was applied aseptically at the centre of the inoculated $\mathrm{MH}$ agar plate, and a $0.5 \mathrm{McFarland}$ standard inoculum of Acinetobacter isolates was streaked from the edge of the imipenem disk (10ug) to the edge of the $\mathrm{MH}$ agar plate. After aerobic incubation at $37^{\circ} \mathrm{C}$ for $18-24 \mathrm{hrs}, \mathrm{MH}$ agar plates were observed for cloverleaf effect or indentation at the intersection of the test bacterium and the E. coli ATCC 25922 control strain within the inhibition zone of the imipenem disc $(10 \mu \mathrm{g})$ [18].

The combined disc test (CDT) was performed as previously described by Yong et al. [19]. Test organism of carbapenem-resistant Acinetobacter with a turbidity of 0.5 McFarland standard was inoculated onto Mueller-Hinton agar plate (Oxoid, UK) [16]. A 0.5M EDTA solution was prepared by dissolving $18.61 \mathrm{~g}$ of disodium EDTA in $100 \mathrm{ml}$ of distilled water and adjusting its $\mathrm{pH}$ to 8.0 by the addition of $\mathrm{NaOH}$ and then autoclaved [19]. Two $10 \mu \mathrm{g}$ imipenem discs (Oxoid, UK) were placed onto the inoculated surface of the $\mathrm{MH}$ agar plate at $20 \mathrm{~mm}$ apart from center to center and $5 \mu \mathrm{l}$ of the prepared $0.5 \mathrm{M}$ EDTA solution was added to one imipenem disc (Oxoid, UK) to obtain a desired concentration of $750 \mu \mathrm{g}$. After $16-18 \mathrm{hrs}$ of aerobic incubation at $37^{\circ} \mathrm{C}$, the inhibition zone displayed around imipenem (Oxoid, UK) and imipenem-EDTA were read and compared. A zone size difference of $\geq 7 \mathrm{~mm}$ was taken as indicative of metallo-betalactamase production as described by Yong et al. [19]. This procedure was repeated to ensure the reproducibility of the result.

2.5. DNA Extraction of MBL Producers of Acinetobacter spp. Bacterial DNA template for PCR assay was extracted by using the whole-cell boiled lysate method [21]. Briefly, four to five colonies of fresh bacterial culture on MacConkey agar were transferred into 500ul of sterile saline in an Eppendorf tube and vortexed briefly, followed by heating at $100^{\circ} \mathrm{C}$ for $10 \mathrm{~min}$. These were then centrifuged at $17000 \mathrm{~g}$ for $5 \mathrm{~min}$, and the supernatants were transferred into fresh Eppendorf tubes to serve as a DNA template for subsequent polymerase chain reaction (PCR). 
TABLE 1: Primers used for the detection of MBL genes.

\begin{tabular}{lccc}
\hline Primer name & Primer sequences & Genes & Size (bp) \\
\hline IMP-R & GGAATAGAGTGGCTTAACTCTC & blaIMP & 232 \\
IMP-F & GTTTAACAAAACAACCACC & blaVIM & 390 \\
VIM-R & TGGTGTTTGGTCGCAAT & & {$[24]$} \\
VIM-F & CGAATGCGCAGCACCAG & blaNDM & 621 \\
NDM-R & CGGAATGGCTCATCACGATC & & {$[24]$} \\
NDM-F & GGTTTGGCGATCTGGTTTTC & & \\
\hline
\end{tabular}

Table 2: Age group, sex , and clinical specimen distribution among Acinetobacter spp.

\begin{tabular}{|c|c|c|c|c|c|c|c|c|}
\hline \multirow{2}{*}{ Sex } & \multicolumn{8}{|c|}{ Age groups } \\
\hline & $<1$ & $1-10$ & $11-20$ & $21-30$ & $31-40$ & $41-50$ & $>50$ & TOTAL (\%) \\
\hline Female & 11 & 0 & 6 & 13 & 8 & 3 & 13 & $54(62.1)$ \\
\hline Male & 5 & 7 & 0 & 4 & 1 & 4 & 12 & $33(37.9)$ \\
\hline Total & 16 & 7 & 6 & 17 & 9 & 7 & 25 & $87(100)$ \\
\hline \multicolumn{9}{|l|}{ Specimen } \\
\hline Aspirate & 1 & - & - & 2 & - & - & - & $3(3.5)$ \\
\hline Ear swab & 5 & - & - & 1 & - & 1 & 2 & $8(9.2)$ \\
\hline Eye swab & 5 & - & - & - & - & - & - & $6(6.9)$ \\
\hline Urine & 1 & 1 & 2 & 6 & 6 & 1 & 8 & $25(28.7)$ \\
\hline Wound swab & 4 & 6 & 4 & 8 & 3 & 5 & 15 & $45(51.7)$ \\
\hline Total & 16 & 7 & 6 & 17 & 9 & 7 & 25 & $87(100)$ \\
\hline
\end{tabular}

2.6. PCR Detection of MBL Encoding Genes of Acinetobacter $s p$. Polymerase chain reaction for MBLs encoding genes was carried out using Eppendorf, master cycler epgradient $S$ (BIORAD, USA). Briefly, multiplex PCR amplification was performed to detect MBL genes (blaIMP, blaVIM, and blaNDM) [22] (Table 1). Each reaction mixture contained $5 \mu \mathrm{l}$ of $4 \mathrm{x}$ PCR buffer, $4 \mathrm{mmol}^{-1} \mathrm{MgCl}_{2}, 2 \mu \mathrm{l}$ of $2 \mathrm{mmol}$ deoxynucleotide triphosphate (dNTPs), $0.5 \mu \mathrm{l}$ of $25 \mathrm{pmol}$ each of oligonucleotide primers (IMP-1, VIM-1, and NDM-1) and $1 \mathrm{U}$ of Taq polymerase (Biolab, New England), and included $2 \mu$ l DNA template. The volume was adjusted with sterile distilled water to give $25 \mu \mathrm{l}$.

The amplification was carried out by an initial denaturation at $95^{\circ} \mathrm{C}$ for $5 \mathrm{~min}$, followed by 35 cycles of denaturation at $94^{\circ} \mathrm{C}$ for $30 \mathrm{sec}$, annealing at $53^{\circ} \mathrm{C}$ for $30 \mathrm{sec}$, extension at $72^{\circ} \mathrm{C}$ for $1 \mathrm{~min}$, and ending with final extension at $72^{\circ} \mathrm{C}$ for $7 \mathrm{~min}$. The PCR products were analysed by electrophoresis using a $1.5 \%$ agarose gel, containing ethidium bromide. Klebsiella pneumoniae NCTC 13443 was used as blaNDM positive control, Pseudomonas aeroginosa NCTC 13437 as blaVIM positive control and nuclease free water was used as negative control.

2.7. Statistical Analysis. Data was entered into Microsoft Excel (2010) database and analyzed descriptively with SPSS version 20.0 (SPSS Inc., Chicago, IL). Frequency tables were used to display numbers, percentages of isolates, antibiotic resistance profiles and other variables. Chi-square $\left(X^{2}\right)$ was used for comparison of any two proportions or percentage; $p$ value $\leq 0.05$ was taken as statistically significant.

\section{Results}

3.1. Acinetobacter Isolates' Demographics and Characteristics. In the present study, out of 2950 Gram-negative bacilli isolated at the bacteriology unit of $\mathrm{KBTH}$ between August 2014 and July 2015, 3.0\% (87) were Acinetobacter isolates. The isolates were from wound swabs [45 (51.7\%)], urine [25 (28.7\%)], ear swabs [8 (9.2\%)], eye swabs $6(6.9 \%)$, and aspirates [3 (3.5\%)] (Table 2). The majority of these isolates were obtained from patients who were $>50$ years of age. Females (62.1\%) had a high prevalence of Acinetobacter isolates compared to the males (37.9\%) (Table 2).

3.2. Antibiotic Susceptibility Pattern of Acinotobacter Spp. Based on the Kirby-Bauer disc diffusion test, 62.1\% (54) of Acinetobacter isolates were multidrug-resistant.

High levels of resistance were observed for ceftazidime (75.9\%), ciprofloxacin (64.4\%), cefotaxime (90.8\%), cotrimoxazole (70.1\%), and meropenem (59.8\%) (Table 3).

3.3. Resistance Patterns of CDT+ve and CDT-ve Isolates. The resistance patterns of CDT positive and CDT negative Acinetobacter isolates are shown in (Table 3 ). Relatively high levels of antibiotic resistance were observed in both combined disc test positive and combined disc test negative Acinetobacter isolates $(p>0.05)$ (Table 3$)$. Amongst CDT positives, there was $100 \%, 100 \%, 100 \%$, and $100 \%$ resistance to ampicillin, cefotaxime, cefuroxime, and ceftazidime, whilst CDT negatives isolates showed resistance of $93.1 \%, 93.1 \%, 93.1 \%$, and $100 \%$, respectively. Table 3 . 
TABle 3: Resistant pattern of Acinetobacter isolates.

\begin{tabular}{lcccc}
\hline Antibiotics & $\begin{array}{c}\text { Resistant } \\
\text { No. }(\%)\end{array}$ & $\begin{array}{c}\text { CDT-ve } \\
\text { Isolates } \\
(23)(\%)\end{array}$ & $\begin{array}{c}\text { CDT-ve } \\
\text { Isolates } \\
(29)(\%)\end{array}$ & $p$ value \\
\hline AMP & $82(94.3)$ & $23(100)$ & $27(93.1)$ & 0.497 \\
AMC & $79(90.8)$ & $22(95.7)$ & $27(93.1)$ & 1 \\
AMK & $22(25.3)$ & $8(34.8)$ & $8(27.6)$ & 0.763 \\
CTX & $75(86.2)$ & $23(100)$ & $27(93.1)$ & 0.497 \\
CXM & $79(90.8)$ & $23(100)$ & $27(93.1)$ & 0.497 \\
CAZ & $66(75.9)$ & $23(100)$ & $29(100)$ & 1 \\
COT & $61(70.1)$ & $17(73.9)$ & $21(72.4)$ & 1 \\
CIP & $56(64.4)$ & $20(87.0)$ & $23(79.3)$ & 0.714 \\
GEN & $63(72.4)$ & $21(95.7)$ & $27(93.1)$ & 0.159 \\
MER & $52(59.8)$ & $23(100)$ & $29(100)$ & 1 \\
NIT & $23(92.0)$ & $14(100)$ & $7(77.8)$ & 0.261 \\
LEV & $59(67.8)$ & $22(95.7)$ & $24(82.7)$ & 0.21 \\
\hline Key:AMP:ampin)
\end{tabular}

Key: AMP: ampicillin; AMC: amoxicillin-clavulanate; AMK: amikacin; CTX: cefotaxime; CXM: cefuroxime, CAZ: ceftazidime, CIP: ciprofloxacin, COT: cotrimoxazole, GEN: gentamicin, MER: meropenem, NIT: nitrofurantoin, LEV: levofloxacin. CDT-ve: combined disc test negative; CDT+ve: combined disc test positive.

3.4. Phenotypic-Based Carbapenemase Detection of Acinetobacter spp. Phenotypic carbapenemase detection was observed in 5.8\% (3/52) meropenem-resistant Acinetobacter by the modified Hodge test (MHT) and 44.2\% (23/52) by the combined disc test (CDT). CDT-positive Acinetobacter isolates were predominant among patients who were $>50$ years (39.12\%), followed by $31-40$ years $(21.7 \%)$ and $<1$ years (13.0\%). CDT-positive Acinetobacter isolates were predominant amongst female patients (65.2\%) (Table 4). Between CDT-positive and CDT-negative isolates, gender distribution was not significant $(p>0.05)$ (Table 4). CDT-positive isolates were mostly obtained from cultures of urine, wound, and ear swabs. Among wound and urine isolates, the difference between CDT-positive and CDT-negative isolates were statistically significant $(p<0.05)$ (Table 4$)$.

3.5. MBL Encoding Genes (blaNDM) among Specimen, Gender, and Age Group. Table 4 shows the distribution of MBL encoding genes in relation to the combined disc test. blaNDM genes were obtained relatively more in female, wound swabs, and among children $<1$ year-old, whilst, lesser blaNDM genes were obtained from male, urine specimen, and adults $>50$ years old.

\section{Discussion}

4.1. Antimicrobial Susceptibility Pattern of Acinetobacter spp. The emergence of multidrug-resistant Acinetobacter isolates is a major concern in hospital settings in many parts of the world [4]. This present study found a high level of resistance to different classes of antibiotics among Acinetobacter isolates. Sixty-two percent (62\%) of the isolates were resistant to at least three or more antimicrobial agents. This is consistent with previous reports from Italy (54\%) and United States $(72 \%)[23,24]$. However, in contrast to our findings, higher
Table 4: Prevalence of blaNDM among specimens, age, sex, and CDT.

\begin{tabular}{lcccc}
\hline Variables & $\begin{array}{c}\text { CDT+ve } \\
(n=23) \\
\text { No. }(\%)\end{array}$ & $\begin{array}{c}\text { CDT-ve } \\
(n=29) \\
\text { No. }(\%)\end{array}$ & $\begin{array}{c}\text { PCR } \\
\text { blaNDM } \\
\text { No. }(\%)\end{array}$ & $p$ value \\
\hline Sex & & & & \\
$\quad$ Female & $15(65.2)$ & $15(51.72)$ & $4(57.1)$ & 0.4026 \\
$\quad$ Male & $8(34.8)$ & $14(48.3)$ & $3(42.9)$ & 0.4026 \\
Specimen & & & & \\
Aspirate & - & $3(10.34)$ & - & 0.2455 \\
Eye swab & - & - & - & \\
Ear swab & $3(13.04)$ & $1(3.44)$ & $1(14.3)$ & 0.3101 \\
Wound swab & $6(26.1)$ & $16(55.2)$ & $4(57.1)$ & $0.0493 *$ \\
Urine & $14(60.9)$ & $9(31.0)$ & $2(28.6)$ & $0.0491 *$ \\
Age group (years) $)$ & & & & \\
$<1$ & $3(13.04)$ & $3(10.34)$ & $3(42.9)$ & 1 \\
$1-10$ & $2(8.7)$ & $4(13.8)$ & - & 0.6821 \\
$11-20$ & $1(4.4)$ & - & - & 0.4423 \\
$21-30$ & $3(13.0)$ & $7(24.14)$ & $1(14.3)$ & 0.4815 \\
$31-40$ & $5(21.7)$ & $3(10.34)$ & $1(14.3)$ & 0.4411 \\
$41-50$ & - & $2(6.9)$ & - & 0.497 \\
$>50$ & $9(39.1)$ & $10(34.5)$ & $2(28.6)$ & 0.778 \\
\hline
\end{tabular}

prevalence is reported in Sudan (97.0\%) and India (91\%) $[25,26]$. The differences in prevalence may be associated with varying dependency on antibiotic usage in different countries [27].

Amongst third-generation cephalosporin in the present study, $75.9 \%$ of the isolates were resistant to ceftazidime. This is consistent with studies in China, India, and Egypt which reported resistance levels of $83.98 \%$ and $84.0 \%$ and $89.0 \%$, respectively $[11,28,29]$. In contrast to the present study, a higher level of resistance to ceftazidime has been reported in Sudan (96\%) and Benin (100\%) [25, 30]. In this study, Acinetobacter resistance to cefotaxime was 90.8\%. This is comparable to studies in Pakistan (99.2\%) and India (100\%) $[25,31]$. The high level of resistance observed to the tested cephalosporins by Acinetobacter spp. may be due to a high level of extended-spectrum- $\beta$-lactamase (ESBLs) induced by selective pressure from broad-spectrum antimicrobial therapy [32].

In the present study, $64.4 \%$ of the isolates were resistant to ciprofloxacin. This is consistent with studies in India (64.0\%) and South Africa (65\%) [29, 32]. Whilst, higher prevalence are reported in Egypt (88.8\%) and Brazil (80\%) $[11,33]$, our findings are higher than studies from Benin $(16 \%)$ and Nigeria $(40.3 \%)$ [30, 34]. In the current study, $67.8 \%$ of Acinetobacter isolates were resistant to levofloxacin. In contrast to our finding, higher prevalence is reported in Mexico $(78.1 \%)$ and China $(82.5 \%)$ [28, 35]. In Ghana, the standard treatment guideline recommends the use of ciprofloxacin for the treatment of urinary tract and bloodstream infections [36]. The high level of resistance observed for fluoroquinolones in the present study is quite worrisome 
and may be due to inappropriate and irrational use of these antibiotics [37].

Acinetobacter isolates were $70.1 \%$ resistant to cotrimoxazole. In contrast to findings in the present study, in India, a slightly higher resistance of $89 \%$ was reported [38]. Resistance to gentamicin was found to be $72.4 \%$. This is comparable with a study in Benin (75\%) [30]. In contrast to this study, lower prevalence is reported in Cameroon $(52.63 \%)$ and South Africa (58\%) [32, 39]. The differences in prevalence may be due to antibiotic policy on the usage of gentamicin for empirical treatment.

Carbapenems have become one of the few selections for the treatment of Acinetobacter and other Gram-negative bacilli infections due to their wider spectrum of antibacterial activity and minimal side effects [4]. The prevalence rate of carbapenem resistance in Acinetobacter baumannii has been found to vary from one country to another [27, 40]. In the present study, 59.8\% Acinetobacter isolates were resistant to carbapenem (meropenem). This is comparable with previous studies in Ghana (66\%), Pakistan (58.9\%) and Nigeria (63.6\%) [13, 31, 41]. Whilst, studies by Hussein et al., [42] and Ren et al., [28] have reported carbapenem (imipenem) resistance of $58.26 \%$ in Iraq and $66 \%$ in China. MorfinOtero et al., [43] and Rajput \& Naik, [44] in Mexico and India have reported a lower carbapenem (imipenem) resistance prevalence of $48 \%$ and $48.57 \%$, respectively. Furthermore, Fattouh and Nasr-Eldin, [10] in Egypt, have reported a higher carbapenem resistance of $71.4 \%$ to Acinetobacter spp. The high resistance to meropenem may be due to the intrinsic ability of Acinetobacter to quickly utilize the efflux pumping mechanism or the capacity to acquire resistant determinants from the environment in response to selective pressure [45].

4.2. Prevalence of $M B L$ Producers in Acinetobacter spp. Among the 52 (59.8\%) meropenem-resistant Acinetobacter isolates, less than $6 \%(3 / 52)$ were positive carbapenemase producers by the modified Hodge test (MHT); although, low sensitivity and specificity of the MHT method in the detection of MBL-producing isolates have been reported by several studies $[7,46]$. The modified Hodge test is a first-line detection method for carbapenemase detection in carbapenem-resistant Enterobacteriaceae [47]. Since, there is no specific phenotypic method phenotypic method recommended for carbapenemase screening and confirmation in Acinetobacter spp. [47]. Different investigators, therefore, employ various detection methods like the combined disc test, double-disc synergy test, and MBL E-test $[12,18,19]$.

The present study used the combined disc test (CDT) for phenotypic MBL detection; this method is specific, sensitive, and easy to perform in a different range of laboratories [19]. The phenotypic prevalence of CDT-positive Acinetobacter isolates was $44.2 \%$. This is similar to studies in South Africa and India which reported a CDT prevalence of $45 \%$ and $44.8 \%$, respectively $[12,48]$. In contrast to findings in this study, a lower CDT prevalence of $34.3 \%$ was reported in Egypt [11]. However, in Iran, 86.8\% of prevalence is reported [49]. The varying MBL prevalence may be due to the different phenotypic detection methods employed by the various investigators in the different countries $[12,18,19]$.
4.3. Prevalence of Metallo-Beta-Lactamase Encoding Genes. Metallo-beta-lactamase-producing Acinetobacter infections have become a public health concern due to few therapeutic choices for the treatment of such infections. MBL-producing Acinetobacter possesses intrinsic potential to acquire and maintain resistance genotypes to different classes of antibiotics (beta-lactams and non-beta-lactams) [46]. Molecular-based techniques are the gold standard for the identification and differentiation of carbapenemase genes [21]. In the present study, multiplex polymerase chain reaction (PCR) was used to evaluate the presence of some MBL encoding genes (blaVIM, blaIMP, and blaNDM) [21]. Among the 23 (44.2\%) CDT-positive Acinetobacter isolates, 7 (8.1\%) haboured MBL encoding gene (blaNDM). However, no blaIMP and blaVIM was detected. This is comparable to a previous study in Ghana that reported 8.1\% carbapenem-resistant Acinetobacter baumannii harbouring blaNDM [13]. Similar studies in Egypt and Kenya have equally reported a prevalence of $19.2 \%$ and $6.25 \%$ bla NDM in MBL-producing Acinetobacter $[50,51]$. The nondetection of blaNDM in $91.9 \%$ of the nonMBL producing Acinetobacter isolates in the present study may be due to the presence of other resistance genes such as blaGIM or blaSIM [21]. The MBL encoding gene (blaNDM) was detected mostly from wound swabs and urine. This is consistent with an earlier study in Ghana [13]. The occurrence of blaNDM gene may be suggestive that the blaNDM gene is one of the common carbapenemase genes circulating among Acinetobacter spp. and other related Gram-negative bacilli in Ghanaian hospitals. Metallo-betalactamase encoding gene (blaNDM) was first identified in a Swedish patient, who was hospitalized in India [52]. The Indian and Pakistan regions have been found to be the primary reserviour for blaNDM genes; since this discovery, blaNDM genes have disseminated to over 40 countries including Kenya, South Africa, Morrocco, Algeria, Iraq, Kuwait, Oman, Israel, the United Kingdom, and the United States [7]. Previously, dissemination of NDM genes was initially attributed to medical tourisms to the India subcontinent [53]. However, recent findings have associated the presence of the blaNDM gene to local spread in the environment $[54,55]$. Metallo-beta-lactamase encoding blaNDM gene presently is a public health menace and infections caused by bacteria carrying these genes are difficult to treat. Furthermore, they have a high propensity for horizontal transfer to neighboring Gram-negative bacilli; hence, the presence of blaNDM gene in Ghanaian hospital is worrisome and calls for prompt detection, surveillance, and strict infection control measures. In conclusion, PCR analysis for blaVIM, blaIMP, and blaNDM showed that less than $9 \%$ of 87 Acinetobacter spp. harboured bla NDM encoding genes. Also, high levels of resistance to multiple antibiotics were found amongst MBL-producing Acinetobacter isolates. The detection of blaNDM amongst MBL-producing-Acinetobacter is a cause for concern; therefore, strict antibiotic usage plan and infection control measures are required to prevent the spread of these resistance genes.

4.4. Strength and Weakness. The sample size was small and represents less than $5 \%$ of the total population of patients 
visiting the health facility (KBTH). This study is the second of its kind in the facility, and we were restricted by the inability to assess other isolates from previous years. A bigger multisectorial survey isolates from patients in major regional hospitals that may incorporate treatment, screening of entire panels of OXA, and MBL encoding genes and other clinical variables are planned pending appropriate funding. Access to medical history was limited; hence, our inability to link our findings to any clinical conditions. Also, there was no follow-up of patients to determine whether the treatment was administered and whether it had been effective. Furthermore, the small number of the isolates where the gene was detected makes it hard to draw solid conclusions.

\section{Data Availability}

The datasets used and/or analysed during the current study are available from the corresponding author on reasonable request.

\section{Ethical Approval}

The study was approved by the Ethics and Protocol Review Committee of the School of Biomedical and Allied Health Sciences (Ethic Identification Number: SBAHSMD/10550758/AA/5A/2016-2017).

\section{Conflicts of Interest}

The authors declare that there is no conflict of interests regarding the publication of this paper.

\section{Acknowledgments}

Our appreciation goes to all the staff of Korle-Bu Teaching Hospital and all patients for their cooperation and support during the various aspects of the study. Our thanks are due to the $31^{\text {st }}$ Euro Global Summit and Expo on Vaccines \& Vaccination and $4^{\text {th }}$ World Congress and Exhibition on Antibiotics and Antibiotic Resistance for abstract poster presentation of manuscript.

\section{References}

[1] A. Y. Peleg, H. Seifert, and D. L. Paterson, "Acinetobacter baumannii: emergence of a successful pathogen," Clinical Microbiology Reviews, vol. 21, no. 3, pp. 538-582, 2008.

[2] P. G. Higgins, C. D. Dammhay, M. Hackel, and H. Seifert, "Global spread of carbapenem-resistant Acinetobacter baumannii," Journal of Antimicrobial Chemotherapy, vol. 65, no. 2, pp. 233-238, 2010.

[3] M. Souli, I. Galaini, and H. Giamarellou, "Emergence of extensively drug-resistant and pandrug resistant Gram negative bacilli in Europe," Eurosurveillance, vol. 13, pp. 1-10, 2008.

[4] K. M. Papp-Wallace, A. Endimiani, M. A. Taracila, and R. A. Bonomo, "Carbapenems: past, present, and future," Antimicrobial Agents and Chemotherapy, vol. 55, no. 11, pp. 49434960, 2011.

[5] World Health Organization, WHO publishes list of bacteria for which New Antibiotics are urgently needed, World Health Orga- nization, Geneva, 2017, http://www.who.int/mediacentre/news/ releases/2017/bacteria-antibiotics-needed/en/.

[6] L. Poirel and P. Nordmann, "Carbapenem resistance in Acinetobacter baumannii: mechanisms and epidemiology," Clinical Microbiology Infection Review, vol. 12, no. 9, pp. 826-836, 2006.

[7] P. Nordmann, T. Naas, and L. Poirel, "Global spread of carbapenemase-producing Enterobacteriaceae," Emerging Infectious Disease, vol. 17, no. 10, pp. 1791-1798, 2011.

[8] R. C. Moldering, "NDM-1 - A Cause for Worldwide Concern," New England Journal of Medicine, vol. 363, no. 25, pp. 2377-2379, 2010.

[9] K. A. Anoar, F. A. Ali, and S. A. Omar, "Detection of metallo$\beta$-lactamase enzymes in some Gram-negative bacteria isolated from burn patients in Sulaimani city Iraq," European Scientific journal, vol. 10, no. 3, pp. 1857-7881, 2014.

[10] M. Fattouh and A. Nasr El-din, "Emergence of carbapenemresistant Acinetobacter baumannii in the intensive care unit in Sohag University Hospital, Egypt," International Journal of Current Microbiology Applied Science, vol. 3, no. 4, pp. 732-744, 2014.

[11] R. A. El-Din, H. El-Bassat, M. El-Bedewy, and H. El-Mohamady, "Prevalence of metallo- $\beta$-lactamse producers among carbapenem-resistant Acinetobacter baunmannii strains isolated from diabetic foot ulcers," African Journal of Microbiology Research, vol. 8, no. 5, pp. 1580-1585, 2015.

[12] M. M. Ehlers, J. M. Hughes, and M. M. Kock, Prevalence of carbapenemases in Acinetobacter baumannii, antibiotic resistant bacteria: a continuous challenge in the new millennium, M. Pana, Ed., pp. 214-246, 2012.

[13] F. S. Codjoe, E. S. Donkor, T. J. Smith, and K. Miller, "Phenotypic and genotypic characterization of carbapenem-resistant gram-negative bacilli pathogens from Hospitals in Ghana," Microbial Drug Resistance, vol. 25, no. 10, pp. 1449-1457, 2019.

[14] KBTH, 2013, Annual report, May 2016, http://www.kbth.gov .gh/.

[15] E. Konemann, S. Allen, and W. Janda, Koneman's Color Atlas and Textbook of Diagnostic Microbiology, Lippincott Williams \& Wilkins, Philadelphia, USA, Sixth edition, 2006.

[16] Clinical and Laboratory Standards Institute (CLSI), Performance standards for antimicrobial susceptibility testing twenty-fifth informational supplement. CLSI document M100$S 25$, Clinical and laboratory standards institute, Wayne, Pennsylvania, USA, 2015.

[17] European Committee on Antimicrobial Susceptibility Testing Breakpoint table for Interpretation of MIC and Zone diameter Version 5.0 valid from 2015-01-01, 2017, May 2016, http://www.eucast.org/ast_of_bacteria/previous_version_of_ documents/.

[18] K. Lee, Y. Chong, H. B. Shin, Y. A. Kim, D. Yong, and J. H. Yum, "Modified Hodge and EDTA-disk synergy tests to screen metallo- $\beta$-lactamase-producing strains of Pseudomonas and Acinetobactet species," Clinical Microbiology and Infection, vol. 7, no. 2, pp. 88-91, 2001.

[19] D. Yong, K. Lee, J. H. Yum, H. B. Shin, G. M. Rossolini, and Y. Chong, "Imipenem-EDTA disk method for differentiation of Metallo- -Lactamase-Producing Clinical isolates of Pseudomonas spp. and Acinetobacter spp," Journal Clinical Microbiology, vol. 40, no. 10, pp. 3798-3801, 2002.

[20] A. P. Magiorakos, A. Srinivasan, R. B. Carey et al., "Multidrugresistant, extensively drug-resistant and pandrug-resistant 
bacteria: an international expert proposal for interim standard definitions for acquired resistance," Clinical Microbiology and Infection, vol. 18, no. 3, pp. 268-281, 2012.

[21] M. Purohit, D. K. Mendiratta, V. S. Deotale, M. Madhan, A. Manoharan, and P. Narang, "Detection of metallo- $\beta$-lactamases producing Acinetobacter baumannii using microbiological assay, disc synergy test and PCR," Indian Journal of Medical Microbiology, vol. 30, no. 4, pp. 456-461, 2012.

[22] L. Poirel, T. R. Walsh, V. Curvillier, and P. Nordamnn, "Multiplex PCR for detection of acquired carbapenemase genes," Diagnostic Microbiology and Infectious Disease, vol. 70, no. 1, pp. 119-123, 2011.

[23] M. A. De Francesco, G. Ravizzola, L. Peroni, C. Bonfanti, and N. Manca, "Prevalence of multidrug-resistant Acinetobacter baumannii and Pseudomonas aeruginosa in an Italian hospital," Journal of Infection and Public Health, vol. 6, no. 3, pp. 179-185, 2013.

[24] L. Dent, R. M. Dana, and P. Siddharth, "Multi-drug resistant Acinetobacter baumannii: a descriptive study in a city hospital," BMC Infectious Diseases, vol. 10, no. 1, 2010.

[25] M. I. Omer, S. A. Gumaa, A. A. Hassan et al., "Prevalence and resistance profile of Acinetobacter baumannii clinical isolates from a private hospital in Khartoum, Sudan," American Journal of Microbiological Research, vol. 3, no. 2, pp. 76-79, 2015.

[26] D. Rynga, M. Shariff, and M. Deb, "Phenotypic and molecular characterization of clinical isolates of Acinetobacter baumannii isolated from Delhi, India," Annals of Clinical Microbiology Antimicrobial, vol. 14, no. 1, p. 40, 2015.

[27] R. A. Bonnin, V. O. Rotimi, M. Al Hubail et al., "Wide dissemination of GES-type carbapenemases in Acinetobacter baunmannii isolates in Kuwait," Antimicrobial agents and chemotherapy, vol. 57, no. 1, pp. 183-188, 2012.

[28] G. Ren, M. Zhou, N. Ding, and Q. Li, “Analysis on distribution features and drug resistance of clinically isolated Acinetobacter baumannii," Expermental and Therapeutic Medicine, vol. 12, no. 3, pp. 1715-1718, 2016.

[29] S. Mohanty, V. Maurya, R. Gaind, and M. Deb, "Phenotypic characterization and colistin susceptibilities of carbapenemresistant of Pseudomonas aeruginosa and Acinetobacter spp," The Journal of Infection in Developing Countries, vol. 7, no. 11, pp. 880-887, 2013.

[30] T. A. Ahoyo, H. S. Bankolé, F. M. Adéoti et al., "Prevalence of nosocomial infections and anti-infective therapy in Benin: results of the first nationwide survey in 2012," Antimicrobial Resistance and Infection Control, vol. 3, no. 1, p. 17, 2014.

[31] M. Sohail, A. Rashid, B. Aslam et al., "Antimicrobial susceptibility of Acinetobacter clinical isolates and emerging antibiogram trends for nosocomial infection management," Revista da Sociedade Brasileira de Medicina Tropical, vol. 49, no. 3, pp. 300-304, 2016.

[32] M. M. Kock, A. N. Bellomo, N. Storm, and M. M. Ehlers, "Prevalence of carbapenem resistance genes in Acinetobacter baumannii isolated from clinical specimens obtained from an academic hospital in South Africa," South African Journal of Epidemiology Infection, vol. 28, no. 1, pp. 28-32, 2015.

[33] R. F. Cereda, H. D. Azevedo, R. Girardello, D. E. Xavier, A. C. Gales, and INVITA-A-CEFTO Brazilian Study Group, "Antimicrobial activity of ceftobiprole against gram-negative and gram-positive pathogens: results from INVITA-A-CEFTO Brazilian study," The Brazilian Journal of Infectious Diseases, vol. 15, no. 4, pp. 339-348, 2011.
[34] I. We, B. A. Adeniyi, and O. O. Soge, "Prevalence of multidrug resistant Acinetobacter baumannii in eight tertiary hospitals in Southwestern Nigeria," New York Science Journal, vol. 7, no. 11, pp. 86-93, 2014.

[35] R. Morfin-Otero, J. C. Tinoco-Favila, H. S. Sader et al., "Resistance trends in gram-negative bacteria: surveillance results from two Mexican hospitals, 2005-2010," BMC Research Notes, vol. 5, no. 1, pp. 277-285, 2012.

[36] $\mathrm{MOH}$, Ministry of Health, Ghana. Standard Treatment Guidelines, 6th edition, , 2010, March 2016, who.int/medicinedocs/ documents/s18015en/s18015en.pdf.

[37] I. N. Okeke, O. A. Aboderin, D. K. Byarugaba, K. K. Ojo, and J. A. Opintan, "Growing problem of multidrug-resistant enteric pathogens in Africa," Emerging Infectious Disease, vol. 13, no. 11, pp. 1640-1646, 2007.

[38] S. S. Somashekara, S. Deepalaxmi, N. Jagannath, and B. Ramesh, "Retrospective analysis of antibiotic resistance pattern to urinary pathogens in a tertiary care hospital in South India," Journal of Basic and Clinical Pharmacy, vol. 5, no. 4, pp. 105-108, 2014.

[39] C. O. Ebongue, E. R. Mengue, J. Nda Mefo'o, M. D. Tsiazok, R. N. Kouassi, and E. N. Bum, "Antimicrobial multiresistance of Acinetobacter baumanii isolated from clinical specimens in Douala (Cameroon)," Journal of Diseases and Medicinal Plants, vol. 1, no. 2, pp. 31-36, 2015.

[40] E. Fonseca, E. Scheidegger, F. S. Freitas, R. Cipriano, and A. C. P. Vicente, "Carbapenem-resistant Acinetobacter baumannii from Brazil: role of carO alleles expression and blaOXA-23 gene," BMC Microbiology, vol. 13, no. 1, p. 245, 2013.

[41] G. Odewale, O. J. Adefioye, J. Ojo, F. A. Adewumi, and O. A. Olowe, "Multidrug resistance ofAcinetobacter baumanniiin Ladoke Akintola University Teaching Hospital, Osogbo, Nigeria," European Journal of Microbiology and Immunology, vol. 6, no. 3, pp. 238-243, 2016.

[42] H. N. Hussein, J. H. F. Al-Mathkhury, and A. M. Sabbah, "Imipenem-resistant Acinetobacter baumannii isolated from patients and hospitals environment in Baghdad," Iraqi Journal of Science, vol. 55, no. 4, pp. 803-812, 2013.

[43] R. Morfín-Otero, M. D. Alcántar-Curiel, M. J. Rocha et al., "Acinetobacter baumannii infections in a tertiary care hospital in Mexico over the past 13 years," Chemotherapy, vol. 59, no. 1, pp. 57-65, 2013.

[44] V. B. Rajput and J. P. Naik, "Carbapenems resistance in gramnegative bacilli isolates from respiratory tract samples of patients in intensive care units," World Journal of Pharmaceutical Research, vol. 4, no. 5, pp. 2495-2505, 2015.

[45] M. Kempf and J. M. Rolain, "Emergence of resistance to carbapenems in Acinetobacter baumannii in Europe: clinical impact and therapeutic options," International Journal of Antimicrobial Agents, vol. 39, no. 2, pp. 105-114, 2012.

[46] D. Doyle, G. Peirano, C. Lascols, T. Lloyd, D. L. Church, and J. D. D. Pitout, "Laboratory detection of Enterobacteriaceae that produce carbapenemases," Journal of Clinical Microbiology, vol. 50, no. 12, pp. 3877-3880, 2012.

[47] Clinical and Laboratory Standards Institute (CLSI), Performance standards for antimicrobial susceptibility testing twentyfirsth informational supplement. CLSI document M100-S21, Clinical and laboratory standards institute, Wayne, Pennsylvania, USA, 2012.

[48] M. N. Moghadam, M. Motamedifar, J. Sarvari, E. S. H. Sedigh, S. M. Mousavi, and F. N. Moghadam, "Emergence of Multidrug 
Resistance and metallo-beta-lactamase producing Acinetobacter baumannii isolated from patients in Shiraz, Iran," Annals of Medical and Health Sciences Research, vol. 6, no. 3, pp. 162167, 2016.

[49] M. Noori, A. Karimi, F. Fallah et al., "High prevalence of metallo-beta-lactamase producing Acinetobacter baumannii isolated from two hospitals of Tehran, Iran," Archived Pediatric Infectious Diseases, vol. 2, no. 3, pp. 1-5, 2014.

[50] G. Revathi, K. L. Siu, P. Lu, and L. Huang, "First report of NDM-1-producing Acinetobacter baumannii in East Africa," International Journal of Infectious Diseases, vol. 17, no. 12, pp. e1255-e1258, 2013.

[51] R. Reem and A. El-Glil, "New Delhi metallo- $\beta$-lactamase 1 (NDM-1) producing Acinetobacter baunmannii in Egyptian hospital," International Journal of Advance Research, vol. 3, no. 4, pp. 470-478, 2015.

[52] D. Yong, M. A. Toleman, C. G. Giske et al., "Characterization of a new metallo- $\beta$-lactamase gene, blaNDM-1, and a novel erythromycin esterase gene carried on a unique genetic structure in Klebsiella pneumoniae sequence type 14 from India," Antimicrobial Agents Chemotherapy, vol. 53, no. 12, pp. 5046-5054, 2009.

[53] K. K. Kumarasamy, M. A. Toleman, T. R. Walsh et al., "Emergence of a new antibiotic resistance mechanism in India, Pakistan, and the UK: a molecular, biological, and epidemiological study," The Lancet Infectious Diseases, vol. 10, no. 9, pp. 597602, 2010.

[54] B. Rimrang, A. Chanawong, A. Lulitanond et al., "Emergence of NDM-1- and IMP-14a-producing Enterobacteriaceae in Thailand," Journal Antimicrobial Chemotherapy, vol. 67, no. 11, pp. 2626-2630, 2012.

[55] J. Yang, Y. Chen, X. Jia et al., "Dissemination and characterization of NDM-1-producing Acinetobacter pittii in an intensive care unit in China," Clinical Microbioloy of Infection, vol. 18, no. 12, pp. E506-E513, 2012. 